\title{
AN $\eta$-APPROXIMATION METHOD FOR NONSMOOTH MULTIOBJECTIVE PROGRAMMING PROBLEMS
}

\author{
TADEUSZ ANTCZAK ${ }^{1}$ \\ (Received 19 November, 2007; revised 12 April, 2008)
}

\begin{abstract}
In this paper, a new approach to a characterization of solvability of a nonlinear nonsmooth multiobjective programming problem with inequality constraints is introduced. A family of $\eta$-approximated vector optimization problems is constructed by a modification of the objective and the constraint functions in the original nonsmooth multiobjective programming problem. The connection between (weak) efficient points in the original nonsmooth multiobjective programming problem and its equivalent $\eta$ approximated vector optimization problems is established under $V$-invexity. It turns out that, in most cases, solvability of a nonlinear nonsmooth multiobjective programming problem can be characterized by solvability of differentiable vector optimization problems.
\end{abstract}

2000 Mathematics subject classification: 90C26, 90C29, 90C46, 49J52.

Keywords and phrases: nonsmooth multiobjective programming, $V$-invex function with respect to $\eta, \eta$-approximated vector optimization problem, (weak) Pareto optimal point.

\section{Introduction}

Over recent years, there has been growing interest among researchers in the study of optimality conditions for multiobjective programming problems, which are also known as vector optimization problems. The study of mathematical techniques developed in order to obtain optimality conditions in multiobjective programming problems is often related to the convexity of all functions constituting these optimization problems $[8,15,17-22]$.

In the past few years, the literature relative to the other families of more general functions used to substitute the convex functions in optimization theory has grown immensely. In 1981, Hanson [11] introduced a new class of generalized convex functions to prove the sufficiency of the Karush-Kuhn-Tucker necessary optimality conditions in nonconvex differentiable optimization problems. Later, Craven [7]

\footnotetext{
${ }^{1}$ Faculty of Mathematics and Computer Science, University of Łódź, Banacha 22, 90-238 Łódź, Poland; e-mail: antczak@math.uni.lodz.pl.

(C) Australian Mathematical Society 2008, Serial-fee code 0334-2700/08
} 
named it a class of invex functions. For differentiable vector functions, Jeyakumar and Mond [12] extended the notion of invexity and they introduced a class of $V$-invex functions. Recently, there has been an increasing interest in developing optimality conditions for nonsmooth multiobjective programming problems involving (locally) Lipschitz functions. For the most part, the study of nonsmooth generalized invexity has been in the context of nondifferentiable nonconvex functions (see, for example, Antczak [5], Egudo and Hanson [9], Giorgi and Guerraggio [10], Kim and Schaible [13], Reiland [16]).

Recently, considerable attention has been given to devising new methods that allow the characterization of solvability of the original multiobjective programming problem by the help of an associated vector optimization problem. Antczak introduced new approaches, the so-called modified objective function method [1] and, as its extension, the so-called $\eta$-approximation method [2], to characterize solvability of differentiable multiobjective programming problems with invex functions. In these methods, optimality conditions for the (weak) Pareto optimality problem were obtained by constructing an equivalent vector minimization problem for the considered differentiable multiobjective programming.

This paper extends the $\eta$-approximation method introduced by Antczak [2] for the characterization of solvability of a differentiable multiobjective programming problem to the nonsmooth case. The main purpose of this paper is to obtain the optimality conditions for (weak) Pareto optimal solutions in nonsmooth multiobjective programming problems with locally Lipschitz functions by using the so-called $\eta$ approximation approach. In this method, for the original nonsmooth multiobjective programming problem, we construct a family of associated vector optimization problems by modifying both the objectives and the constraints at an arbitrary but fixed point $(\bar{x}, \bar{\xi}, \bar{\zeta})$, where $\bar{x}$ is a feasible solution for the considered vector optimization problem, and $\bar{\xi}$ and $\bar{\zeta}$ are fixed Clarke's generalized gradients of the objective function and the inequality constraint function at $\bar{x}$, respectively. This construction depends heavily on results proved in this paper, which connect the (weakly) efficient points of the original nondifferentiable multiobjective programming problem to the (weakly) efficient points of the modified vector minimization problem. This equivalence between these vector optimization problems is established under the assumption that all functions constituting the original multiobjective programming problem are locally Lipschitz $V$-invex at $\bar{x}$ on the set of all feasible solutions with respect to the same function $\eta$ and with respect to, not necessarily, the same functions $\alpha$. In this way, we obtain a family of the so-called associated $\eta$-approximated vector optimization problems with the same (weak) Pareto optimal solution $\bar{x}$ and the same optimal value as in the original nonsmooth multiobjective programming problem. Furthermore, the associated $\eta$-approximated vector optimization problems have, in general, simpler forms than the original nonlinear nonsmooth multiobjective programming problem and, therefore, they are easier to solve (in most cases, they are smooth and linear (or convex)). In this paper, this result is illustrated by suitable examples of nonlinear nonsmooth multiobjective programming problems. 


\section{Preliminaries}

The following convention for equalities and inequalities will be used throughout the paper.

For any $x=\left(x_{1}, x_{2}, \ldots, x_{n}\right)^{T}, y=\left(y_{1}, y_{2}, \ldots, y_{n}\right)^{T}$, we define:

(i) $x=y$ if and only if $x_{i}=y_{i}$ for all $i=1,2, \ldots, n$;

(ii) $x<y$ if and only if $x_{i}<y_{i}$ for all $i=1,2, \ldots, n$;

(iii) $x \leqq y$ if and only if $x_{i} \leq y_{i}$ for all $i=1,2, \ldots, n$;

(iv) $x \leq y$ if and only if $x \leqq y$ and $x \neq y$.

Definition 2.1 [6]. If $f: R^{n} \rightarrow R$ is a locally Lipschitz function at $x \in R^{n}$, the generalized derivative (in the sense of Clarke) $f$ at $x \in R^{n}$ in the direction $v \in R^{n}$, denoted by $f^{0}(x ; v)$, is given by

$$
f^{0}(x ; v)=\limsup _{\substack{y \rightarrow x \\ \lambda \downarrow 0}} \frac{f(y+\lambda v)-f(y)}{\lambda} .
$$

Definition 2.2 [6]. Clarke's generalized gradient of $f$ at $x \in R^{n}$, denoted by $\partial f(x)$, is defined as follows:

$$
\partial f(x)=\left\{\xi \in R^{n} \mid f^{0}(x ; v) \geq \xi^{T} v \forall v \in R^{n}\right\} .
$$

REMARK 2.3. It follows that, for any $v \in R^{n}$,

$$
f^{0}(x ; v)=\max \left\{\xi^{T} v \mid \xi \in \partial f(x)\right\} .
$$

In this section, in terms of the Clarke subdifferential, we give a definition of locally Lipschitz $V$-invex functions introduced by Egudo and Hanson [9].

Definition 2.4. Let $X$ be a nonempty subset of $R^{n}$. A vector function $f: X \rightarrow$ $R^{k}$ is said to be (locally Lipschitz) $V$-invex at $u \in X$ on $X$ (with respect to $\eta$ and $\left.\alpha:=\left(\alpha_{1}, \ldots, \alpha_{k}\right)\right)$ if there exist functions $\eta: X \times X \rightarrow R^{n}$ and $\alpha_{i}: X \times X \rightarrow$ $R_{+} \backslash\{0\}, i=1, \ldots, k$, such that, for all $x \in X$, the inequality

$$
f_{i}(x)-f_{i}(u) \geqq \alpha_{i}(x, u) \xi^{T} \eta(x, u), \quad i=1, \ldots, k,
$$

holds for each $\xi_{i} \in \partial f_{i}(u)$. If the inequality (2.2) holds for any $u \in X$, then $f$ is (locally Lipschitz) $V$-invex with respect to $\eta$ and $\alpha$ on $X$.

Each function $f_{i}$ satisfying (2.2) is said to be (locally Lipschitz) $\alpha_{i}$-invex with respect to $\eta$ on $X$.

We consider the multiobjective programming problem

$$
\begin{aligned}
& V \text {-minimize } f(x):=\left(f_{1}(x), \ldots, f_{k}(x)\right) \\
& \text { subject to } g(x):=\left(g_{1}(x), \ldots, g_{m}(x)\right) \leqq 0,
\end{aligned}
$$


where $f: X \rightarrow R^{k}$ and $g: X \rightarrow R^{m}$ are locally Lipschitz functions on a nonempty open set $X \subset R^{n}$. Note here that the symbol ' $V$-minimize' stands for vector minimization (thus the weak Pareto optimal solution or the Pareto optimal solution).

Let

$$
D:=\left\{x \in X \mid g_{j}(x) \leqq 0, j \in J=\{1, \ldots, m\}\right\}
$$

denote the set of all feasible solutions of (VP). Furthermore, we denote the set of active constraints at the given feasible point $\bar{x} \in D$ as follows:

$$
J(\bar{x}):=\left\{j \in J \mid g_{j}(\bar{x})=0\right\} .
$$

For such multicriterion optimization problems, the solution is defined in terms of a (weak) Pareto optimal solution ((weakly) efficient solution) in the following sense.

Definition 2.5. A feasible point $\bar{x}$ is said to be a Pareto optimal solution (efficient solution) for (VP) if and only if there exists no $x \in D$ such that

$$
f(x) \leq f(\bar{x}) .
$$

Definition 2.6. A feasible point $\bar{x}$ is said to be a weak Pareto optimal solution (weakly efficient solution, weak minimum) for (VP) if and only if there exists no $x \in D$ such that

$$
f(x)<f(\bar{x}) .
$$

It is well known (see, for example, [10, 13, 14]) that the Karush-Kuhn-Tucker conditions are necessary for optimality in such vector optimization problems under the assumption that a suitable constraint qualification is fulfilled.

In this paper, we use the following constraint qualification.

At a point $\bar{x} \in D$, let us define

$$
\Omega:= \begin{cases}\left\{v \in R^{n} \mid g_{j}^{0}(\bar{x} ; v)<0 \text { for any } j \in J(\bar{x})\right\} & \text { if } J(\bar{x}) \neq \varnothing, \\ R^{n} & \text { if } J(\bar{x})=\varnothing .\end{cases}
$$

Constraint QualificAtion (CQ): At a point $\bar{x} \in D$, it holds that $\Omega \neq \varnothing$.

TheOREM 2.7. Let $\bar{x}$ be a (weak) Pareto optimal solution in problem (VP) and Constraint Qualification (CQ) be satisfied at $\bar{x}$. Then there exist $\bar{\lambda} \in R^{k}$ and $\bar{\mu} \in R^{m}$ such that

$$
\begin{gathered}
0 \in \sum_{i=1}^{k} \bar{\lambda}_{i} \partial f_{i}(\bar{x})+\sum_{j=1}^{m} \bar{\mu}_{j} \partial g_{j}(\bar{x}), \\
\bar{\mu}_{j} g_{j}(\bar{x})=0, \quad j=1, \ldots, m, \\
\bar{\lambda} \geq 0, \quad \bar{\mu} \geqq 0 .
\end{gathered}
$$


Proof. Assume that $\bar{x} \in D$ is a (weak) Pareto optimal solution in the considered nonlinear multiobjective programming problem (VP). Hence, by the Fritz John necessary optimality conditions of Clarke (see [6, Theorem 6.1.3]), there exist $\bar{\lambda} \in R_{+}^{k}$ and $\bar{\mu} \in R_{+}^{m}$, not all simultaneously zero, such that the conditions (2.3) and (2.4) are fulfilled. By elementary calculus of support functions, (2.3) yields

$$
\sum_{i=1}^{k} \bar{\lambda}_{i} f_{i}^{0}(\bar{x} ; v)+\sum_{j=1}^{m} \bar{\mu}_{j} g_{j}^{0}(\bar{x} ; v) \geqq 0 \quad \forall v \in R^{n} .
$$

To prove the generalized Karush-Kuhn-Tucker necessary optimality conditions (2.3)(2.5) for problem (VP) one must have the vector $\bar{\lambda} \neq 0$.

We proceed by contradiction. Suppose that $\bar{\lambda}=0$. Thus,

$$
\sum_{j \in J(\bar{x})} \bar{\mu}_{j} g_{j}^{0}(\bar{x} ; v) \geqq 0 \quad \forall v \in R^{n} .
$$

By assumption, $\Omega \neq 0$. This means that, for all $j \in J(\bar{x})$,

$$
g_{j}^{0}(\bar{x} ; v)<0 \quad \forall v \in R^{n} .
$$

From the Fritz John necessary optimality conditions of Clarke, it follows that $\bar{\mu} \neq 0$, since we have assumed that $\bar{\lambda}=0$. Then, by (2.7), we obtain the inequality

$$
\sum_{j \in J(\bar{x})} \bar{\mu}_{j} g_{j}^{0}(\bar{x} ; v)<0 \quad \forall v \in \Omega \subset R^{n},
$$

which contradicts (2.6).

However, for the nonlinear multiobjective programming problem (VP) considered, the so-called Generalized Slater's Constraint Qualification (GSCQ) can also be formulated. Now, we introduce this constraint qualification as follows.

Generalized Slater's Constraint Qualification (GSCQ). For problem (VP), assume that there exists a point $\tilde{x} \in D$ such that $g_{j}(\tilde{x})<0, j \in J(\bar{x})$, and, moreover, the constraint functions $g_{j}, j \in J(\bar{x})$, are $\beta_{j}$-invex at $\bar{x}$ on $D$ with respect to the same function $\eta$.

REMARK 2.8. It is not difficult to see that if we assume GSCQ then, at the point $\bar{x} \in D$, it holds that $\Omega \neq \varnothing$. However, it follows from the formulation of GSCQ, the constraint functions $g_{j}, j \in J(\bar{x})$, should also be assumed to be $\beta_{j}$-invex at $\bar{x}$ on $D$ with respect to the same function $\eta$.

\section{An equivalent vector optimization problem and optimality conditions}

Let $\bar{x}$ be the given feasible solution in the original nonsmooth multiobjective programming problem (VP) and, moreover, $\bar{\xi}$ and $\bar{\zeta}$ be Clarke's generalized gradients of the objective function $f$ and the constraint function $g$ in (VP) at $\bar{x}$, respectively. We consider a family of $\eta$-approximated vector optimization problems $\left(\operatorname{VP}_{\eta}(\bar{x}, \bar{\xi}, \bar{\zeta})\right)$ given by 
$V$-minimize $\left(f_{1}(\bar{x})+\bar{\xi}_{1}^{T} \eta(x, \bar{x}), \ldots, f_{k}(\bar{x})+\bar{\xi}_{k}^{T} \eta(x, \bar{x})\right)$ subject to $g_{j}(\bar{x})+\bar{\zeta}_{j}^{T} \eta(x, \bar{x}) \leqq 0, \quad j=1, \ldots, m$,

where $f, g, X$ are defined as in (VP), $\bar{\xi}_{i}, i=1, \ldots, k, \bar{\zeta}_{j}, j=1, \ldots, m$, are Clarke's generalized gradients of $f_{i}, i=1, \ldots, k$, and $g_{j}, j=1, \ldots, m$, at $\bar{x}$, respectively, that is, $\bar{\xi}_{i} \in \partial f_{i}(\bar{x}), i=1, \ldots, k, \bar{\zeta}_{j} \in \partial g_{j}(\bar{x}), j=1, \ldots, m$, and $\eta$ is a vector-valued function defined by $\eta: X \times X \rightarrow R^{n}$. Throughout this paper we will assume that $\eta$ satisfies the following condition: $\eta(x, \bar{x}) \neq 0$ for any $x \in X$ such that $x \neq \bar{x}$.

Let

$$
D(\bar{x}, \bar{\zeta}):=\left\{x \in X \mid g_{j}(\bar{x})+\bar{\zeta}_{j}^{T} \eta(x, \bar{x}) \leqq 0, j=1, \ldots, m\right\}
$$

denote the set of all feasible solutions of $\left(\operatorname{VP}_{\eta}(\bar{x}, \bar{\xi}, \bar{\zeta})\right)$.

First, we prove that a (weak) Pareto optimal solution $\bar{x}$ in the multiobjective programming problem (VP) is also (weak) Pareto optimal in its associated $\eta$ approximated vector optimization problem $\left(\operatorname{VP}_{\eta}(\bar{x}, \bar{\xi}, \bar{\zeta})\right)$, if the Clarke's generalized gradients $\bar{\xi}$ and $\bar{\zeta}$ of the objective function $f$ and the constraint function $g$ at $\bar{x}$, respectively, satisfy the Karush-Kuhn-Tucker optimality conditions.

Theorem 3.1. Let $\bar{x}$ be a (weak) Pareto optimal solution in (VP) and the $C Q$ be fulfilled at $\bar{x}$. Furthermore, assume that the generalized Karush-Kuhn-Tucker necessary optimality conditions (2.3)-(2.5) are satisfied at $\bar{x}$ with the Lagrange multipliers $\bar{\lambda} \in R^{k}$ and $\bar{\mu} \in R^{m}$ (with $\bar{\lambda}>0$ in the case when $\bar{x}$ is a Pareto optimal solution in (VP)). If $\eta$ satisfies $\eta(\bar{x}, \bar{x})=0$, then $\bar{x}$ is also (weak) Pareto optimal in $\left(\operatorname{VP}_{\eta}(\bar{x}, \bar{\xi}, \bar{\zeta})\right)$, where $\bar{\xi}=\left(\bar{\xi}_{1}, \ldots, \bar{\xi}_{k}\right)^{T} \in \partial f(\bar{x})$ and $\bar{\zeta}=\left(\bar{\zeta}_{1}, \ldots, \bar{\zeta}_{m}\right)^{T} \in \partial g(\bar{x})$ are Clarke's generalized gradients of $f$ and $g$ at $\bar{x}$, respectively, satisfying the generalized Karush-Kuhn-Tucker necessary optimality conditions (2.3)-(2.5).

Proof. Assume that $\bar{x}$ is a Pareto optimal solution in (VP) and CQ is fulfilled at $\bar{x}$. Then there exist the Lagrange multipliers $\bar{\lambda} \in R^{k}$ and $\bar{\mu} \in R^{m}$ such that the KarushKuhn-Tucker conditions (2.3)-(2.5) are satisfied.

We proceed by contradiction. Suppose that $\bar{x}$ is not a Pareto optimal solution in $\left(\operatorname{VP}_{\eta}(\bar{x}, \bar{\xi}, \bar{\zeta})\right)$. This implies that there exists $\tilde{x}$ which is feasible for $\left(\operatorname{VP}_{\eta}(\bar{x}, \bar{\xi}, \bar{\zeta})\right)$ such that

$$
f(\bar{x})+\bar{\xi} \eta(\tilde{x}, \bar{x}) \leq f(\bar{x})+\bar{\xi} \eta(\bar{x}, \bar{x}),
$$

where, as follows from the definition of $\left(\operatorname{VP}_{\eta}(\bar{x}, \bar{\xi}, \bar{\zeta})\right), \bar{\xi} \in \partial f(\bar{x})$. By assumption, $\eta(\bar{x}, \bar{x})=0$. Then,

$$
\bar{\xi} \eta(\widetilde{x}, \bar{x}) \leq 0
$$

From the assumption, $\bar{\lambda}>0$. Thus,

$$
\bar{\lambda}^{T}[\bar{\xi} \eta(\tilde{x}, \bar{x})]<0 .
$$


By $\tilde{x} \in D(\bar{x}, \bar{\zeta})$ and $\bar{\mu} \geqq 0$,

$$
\bar{\mu}^{T}[g(\bar{x})+\bar{\zeta} \eta(\tilde{x}, \bar{x})] \leqq 0 .
$$

Using the Karush-Kuhn-Tucker optimality condition (2.4), we obtain

$$
\bar{\mu}^{T}[\bar{\zeta} \eta(\tilde{x}, \bar{x})] \leqq 0,
$$

where, as follows from the definition of a nonsmooth vector optimization problem, $\zeta \in \partial g(\bar{x})$. By (3.9) and (3.10), we get the inequality

$$
\left[\bar{\lambda}^{T} \bar{\xi}+\bar{\mu}^{T} \bar{\zeta}\right] \eta(\tilde{x}, \bar{x})<0,
$$

which contradicts the generalized Karush-Kuhn-Tucker necessary optimality condition (2.3). Hence, $\bar{x}$ is weak Pareto optimal in $\left(\operatorname{VP}_{\eta}(\bar{x}, \bar{\xi}, \bar{\zeta})\right)$.

The proof for weak Pareto optimality is similar, but it should be taken into account that the Lagrange multiplier $\bar{\lambda}$ associated with the objective function $f$ satisfies the relation $\bar{\lambda} \geq 0$.

REMARK 3.2. Note that we have established Theorem 3.1 without any assumption regarding to which class of generalized invex functions the functions involved in problems (VP) and $\left(\operatorname{VP}_{\eta}(\bar{x}, \bar{\xi}, \bar{\zeta})\right)$ belong. However, if we assume that the GSCQ is fulfilled at a (weak) Pareto optimal solution $\bar{x}$ in problem (VP), then the constraint functions $g_{j}, j \in J(\bar{x})$, should be assumed to be $\beta_{j}$-invex at $\bar{x}$ on $D$ with respect to the same function $\eta$.

REMARK 3.3. As follows from Theorem 3.1, if $\bar{x}$ is a (weak) Pareto optimal solution in the nonsmooth multiobjective programming problem (VP) then it is also a (weak) Pareto optimal solution in its $\eta$-approximated vector optimization problem $\left(\operatorname{VP}_{\eta}(\bar{x}, \bar{\xi}, \bar{\zeta})\right)$, where $\bar{\xi}=\left(\bar{\xi}_{1}, \ldots, \bar{\xi}_{k}\right)^{T} \in \partial f(\bar{x})$ and $\bar{\zeta}=\left(\bar{\zeta}_{1}, \ldots, \zeta_{m}\right)^{T} \in \partial g(\bar{x})$ are Clarke's generalized gradients of $f$ and $g$ at $\bar{x}$, respectively, satisfying the generalized Karush-Kuhn-Tucker necessary optimality conditions (2.3)-(2.5). This means that the nonsmooth multiobjective programming problem (VP) is not equivalent to each $\eta$-approximated vector optimization problem $\left(\operatorname{VP}_{\eta}(\bar{x}, \bar{\xi}, \bar{\zeta})\right)$ belonging to a family of all $\eta$-approximated vector optimization problems defined at the given point $(\bar{x}, \bar{\xi}, \bar{\zeta})$. We illustrate this result in the next example.

EXAMPLE 3.4. We consider the following nonlinear nonsmooth multiobjective programming problem:

$$
\begin{aligned}
& f(x)=(|x|,|x|) \rightarrow \min , \\
& g(x)=1-e^{x} \leqq 0 .
\end{aligned}
$$

Note that $D=\{x \in R \mid x \geqq 0\}$ and $\bar{x}=0$ is a Pareto optimal point in the considered nonsmooth multiobjective programming problem (VP1). Let $\eta: R \times R \rightarrow R$ be defined by

$$
\eta(x, \bar{x})=x-\bar{x}
$$


and, moreover, $\bar{\xi}$ and $\bar{\zeta}$ be Clarke's generalized gradients of $f$ and $g$ at $\bar{x}$, that is, $\bar{\xi} \in \partial f(\bar{x})=\left\{\left[\xi_{1}, \xi_{2}\right]^{T} \mid-1 \leqq \xi_{1} \leqq 1 \wedge-1 \leqq \xi_{2} \leqq 1\right\}$ and $\bar{\zeta} \in \partial g(\bar{x})=\{-1\}$. We set $\bar{\xi}=\left[\bar{\xi}_{1}, \bar{\xi}_{2}\right]^{T}=[-1,-1]^{T}$ and $\overline{\bar{\zeta}}=-1$, and then we obtain the following $\eta$-approximated vector optimization problem $\left(\operatorname{VP} 1_{\eta}(\bar{x}, \bar{\xi}, \bar{\zeta})\right)$ :

$$
\begin{gathered}
(-x,-x) \rightarrow \min \\
-x \leqq 0 .
\end{gathered}
$$$$
\left(\operatorname{VP} 1_{\eta}(\bar{x}, \bar{\xi}, \bar{\zeta})\right)
$$

It is not difficult to see that $\bar{x}=0$ is not a Pareto optimal point in this $\eta$-approximated vector optimization problem $\left(\operatorname{VP}_{\eta}(\bar{x}, \bar{\xi}, \bar{\zeta})\right)$. This follows from the fact that there do not exist Lagrange multipliers $\bar{\lambda} \in R^{k}$ and $\bar{\mu} \in R^{m}$ such that the generalized KarushKuhn-Tucker necessary optimality conditions (2.3)-(2.5) are satisfied with these Clarke's generalized gradients $\bar{\xi}$ and $\bar{\zeta}$.

REMARK 3.5. The hypothesis of Theorem 3.1, that the Lagrange multiplier $\bar{\lambda}$ associated with the objective function $f$ satisfies the condition $\bar{\lambda}>0$ in the case when $\bar{x}$ is a Pareto optimal solution in (VP), is essential and it cannot be omitted. We consider again the nonlinear nonsmooth multiobjective programming problem (VP) from Example 3.4. Note that $\bar{x}=0$ is a Pareto optimal point in the considered nonsmooth multiobjective programming problem (VP) and Clarke's generalized gradients of $f$ and $g$ at $\bar{x}$ are as follows: $\partial f(\bar{x})=\left\{\left[\xi_{1}, \xi_{2}\right]^{T} \mid-1 \leqq \xi_{1} \leqq 1 \wedge-1 \leqq\right.$ $\left.\xi_{2} \leqq 1\right\}$ and $\partial g(\bar{x})=\{-1\}$. We set $\bar{\xi}=\left[\bar{\xi}_{1}, \bar{\xi}_{2}\right]^{T}=[-1,0]^{T}$ and $\bar{\zeta}=-1$, and then we obtain the following $\eta$-approximated vector optimization problem $\left(\operatorname{VP} 1_{\eta}(\bar{x}, \bar{\xi}, \bar{\zeta})\right)$ :

$$
\begin{gathered}
(-x, 0) \rightarrow \min \\
-x \leqq 0 .
\end{gathered}
$$$$
\left(\operatorname{VP} 1_{\eta}(\bar{x}, \bar{\xi}, \bar{\zeta})\right)
$$

Note that in this case, the generalized Karush-Kuhn-Tucker necessary optimality conditions (2.3)-(2.5) are fulfilled only with the Lagrange multiplier $\bar{\lambda}=\left[\bar{\lambda}_{1}, \bar{\lambda}_{2}\right]^{T}$, where $\bar{\lambda}_{1}=0$. Hence, the hypothesis $\bar{\lambda}>0$ of Theorem 3.1 is not satisfied. It is not difficult to see that $\bar{x}=0$ is not a Pareto optimal point in this vector optimization problem.

Now, under a suitable $V$-invexity assumption with respect to the same function $\eta$, but with respect to, not necessarily, the same function $\alpha$, imposed on the objective function $f$ and the constraint function $g$, we establish that a (weak) Pareto optimal solution $\bar{x}$ in an $\eta$-approximated vector optimization problem $\left(\operatorname{VP}_{\eta}(\bar{x}, \bar{\xi}, \bar{\zeta})\right)$ is also (weak) Pareto optimal in the original multiobjective programming problem (VP). To prove this result we also assume that $\eta$ satisfies the condition $\eta(\bar{x}, \bar{x})=0$.

TheOrem 3.6. Let $\bar{x}$ be a (weak) Pareto optimal solution in $\left(\operatorname{VP}_{\eta}(\bar{x}, \bar{\xi}, \bar{\zeta})\right)$ and $\eta(\bar{x}, \bar{x})=0$. Furthermore, assume that $f$ is $V$-invex at $\bar{x}$ on $D$ with respect to $\eta$ and $\alpha$ and $g$ is $V$-invex at $\bar{x}$ on $D$ with respect to the same function $\eta$ and with respect to $\beta$, where, for all $j \notin J(\bar{x}), \beta_{j}(x, \bar{x}) \geqq 1$ for all $x \in D$. Then $\bar{x}$ is also (weak) Pareto optimal in (VP). 
Proof. By assumption, $g$ is $V$-invex at $\bar{x}$ on $D$ with respect to $\eta$ and $\beta$. Then, by Definition 2.4, the inequalities

$$
\begin{array}{ll}
g_{j}(x) \geqq \beta_{j}(x, \bar{x}) \zeta_{j}^{T} \eta(x, \bar{x}), & j \in J(\bar{x}), \\
g_{j}(x) \geqq g_{j}(\bar{x})+\beta_{j}(x, \bar{x}) \zeta_{j}^{T} \eta(x, \bar{x}), & j \notin J(\bar{x}),
\end{array}
$$

hold for each $\zeta_{j} \in \partial g_{j}(\bar{x})$ for $j \in J$ and for all $x \in D$. Hence, they are satisfied for $\zeta_{j}=\bar{\zeta}_{j} \in \partial g_{j}(\bar{x})$ for $j \in J$. From Definition 2.4, it follows that $\beta_{j}(x, \bar{x})>0$ for all $x \in D$. Dividing both sides of (3.12) by $\beta_{j}(x, \bar{x})$, we obtain

$$
\frac{1}{\beta_{j}(x, \bar{x})} g_{j}(x) \geqq \frac{1}{\beta_{j}(x, \bar{x})} g_{j}(\bar{x})+\bar{\zeta}_{j}^{T} \eta(x, \bar{x}), \quad j \notin J(\bar{x}) .
$$

By assumption, for all $j \notin J(\bar{x}), \beta_{j}(x, \bar{x}) \geqq 1$ for all $x \in D$. Since $g_{j}(\bar{x})<0$ for all $j \notin J(\bar{x})$, then

$$
\frac{1}{\beta_{j}(x, \bar{x})} g_{j}(\bar{x})+\bar{\zeta}_{j}^{T} \eta(x, \bar{x}) \geqq g_{j}(\bar{x})+\bar{\zeta}_{j}^{T} \eta(x, \bar{x}), \quad j \notin J(\bar{x}) .
$$

Thus, by (3.13) and (3.14),

$$
\frac{1}{\beta_{j}(x, \bar{x})} g_{j}(x) \geqq g_{j}(\bar{x})+\bar{\zeta}_{j}^{T} \eta(x, \bar{x}), \quad j \notin J(\bar{x}) .
$$

Then, for any $x \in D$, the inequalities (3.11) and (3.15) imply, respectively,

$$
\begin{array}{ll}
0 \geqq \beta_{j}(x, \bar{x}) \bar{\zeta}_{j}^{T} \eta(x, \bar{x}), \quad j \in J(\bar{x}), \\
0 \geqq g_{j}(\bar{x})+\bar{\zeta}_{j}^{T} \eta(x, \bar{x}), \quad j \notin J(\bar{x}),
\end{array}
$$

where $\bar{\zeta}_{j} \in \partial g_{j}(\bar{x})$ for $j \in J$. From Definition 2.4, it follows that $\beta_{j}(x, \bar{x})>0$ for all $x \in D$. Dividing both sides of (3.16) by $\beta_{j}(x, \bar{x})$, we obtain

$$
0 \geqq \bar{\zeta}_{j}^{T} \eta(x, \bar{x}), \quad j \in J(\bar{x}) .
$$

Since $g_{j}(\bar{x})=0$ for each $j \in J(\bar{x})$, then (3.18) gives

$$
0 \geqq g_{j}(\bar{x})+\bar{\zeta}_{j}^{T} \eta(x, \bar{x}), \quad j \in J(\bar{x}) .
$$

Therefore, by (3.17) and (3.18), it follows that, for all $x \in D$,

$$
0 \geqq g_{j}(\bar{x})+\bar{\zeta}_{j}^{T} \eta(x, \bar{x}), \quad j \in J,
$$

where $\bar{\zeta}_{j} \in \partial g_{j}(\bar{x})$ for $j \in J$. Hence, any feasible solution in (VP) is also feasible in $\left(\operatorname{VP}_{\eta}(\bar{x}, \bar{\xi}, \bar{\zeta})\right)$, that is, $D \subset D(\bar{x}, \bar{\zeta})$. Let $\bar{x}$ be Pareto optimal in $\left(\operatorname{VP}_{\eta}(\bar{x}, \bar{\xi}, \bar{\zeta})\right)$. Then, by Definition 2.5 ,

$$
\nexists x \in D(\bar{x}, \bar{\zeta}), \quad f(\bar{x})+\bar{\xi} \eta(x, \bar{x}) \leq f(\bar{x})+\bar{\xi} \eta(\bar{x}, \bar{x}) .
$$


From $D \subset D(\bar{x}, \bar{\zeta})$ it follows that

$$
\nexists x \in D, \quad f(\bar{x})+\bar{\xi} \eta(x, \bar{x}) \leq f(\bar{x})+\bar{\xi} \eta(\bar{x}, \bar{x}) .
$$

By assumption, $\eta(\bar{x}, \bar{x})=0$. Thus, (3.19) yields

$$
\nexists x \in D, \quad \bar{\xi} \eta(x, \bar{x}) \leq 0 .
$$

We proceed by contradiction. Suppose that $\bar{x}$ is not Pareto optimal in (VP). Then by Definition 2.5 there exists $\tilde{x} \in D$ such that

$$
f(\tilde{x}) \leq f(\bar{x}) .
$$

By assumption $f$ is $V$-invex at $\bar{x}$ on $D$ with respect to $\eta$ and $\alpha$. Then by Definition 2.4, it follows that, for any $i=1, \ldots, k$, the inequality

$$
f_{i}(x)-f_{i}(\bar{x}) \geqq \alpha_{i}(x, \bar{x}) \xi_{i}^{T} \eta(x, \bar{x})
$$

holds for all $x \in D$ and each $\xi_{i} \in \partial f_{i}(\bar{x}), i=1, \ldots, k$. Hence, it is also satisfied for $x=\tilde{x}$ and $\xi_{i}=\bar{\xi}_{i}, i=1, \ldots, k$. Thus, by (3.21),

$$
\alpha_{i}(\tilde{x}, \bar{x}) \bar{\xi}_{i}^{T} \eta(\tilde{x}, \bar{x}) \leqq 0,
$$

but, for at least one $i \in J$,

$$
\alpha_{i}(\tilde{x}, \bar{x}) \bar{\xi}_{i}^{T} \eta(\tilde{x}, \bar{x})<0 .
$$

By Definition 2.4, it follows that, for any $i=1, \ldots, k, \alpha_{i}(\tilde{x}, \bar{x})>0$. Then, by (3.22) and (3.23),

$$
\exists \tilde{x} \in D, \quad \bar{\xi}^{T} \eta(\tilde{x}, \bar{x}) \leq 0,
$$

which contradicts (3.20). Hence, the conclusion of the theorem is proved.

The proof for $\bar{x}$ to be a weak Pareto optimal solution is similar.

In view of Theorem 3.1 and Theorem 3.6, if we assume that the CQ (or GSCQ) is satisfied at $\bar{x}, f$ and $g$ satisfy suitable $V$-invex type conditions at $\bar{x}$ on the set of all feasible solutions $D$ with respect to the same function $\eta$ and with respect to, not necessarily, the same function $\alpha$, and, moreover, $\eta$ satisfies the relation $\eta(\bar{x}, \bar{x})=0$, then the considered nonsmooth multiobjective programming problem (VP) and its $\eta$ approximated vector optimization problem $\left(\operatorname{VP}_{\eta}(\bar{x}, \bar{\xi}, \bar{\zeta})\right)$ are equivalent in the sense discussed above.

Now, we give an example of a nonlinear multiobjective programming problem (VP), for which the $\eta$-approximation approach is used to find a Pareto optimal solution.

EXAMPLE 3.7. We consider the following nonlinear nonsmooth multiobjective programming problem:

$$
\begin{aligned}
f(x)= & \left(x e^{|x-1|}, \arctan |x|\right) \rightarrow \min \\
& g_{1}(x)=1-e^{x} \leqq 0 \\
& g_{2}(x)=|x|-2 \leqq 0
\end{aligned}
$$


Note that $D=\{x \in R \mid 0 \leqq x \leqq 2\}$ and $\bar{x}=0$ is a Pareto optimal point in the considered nonlinear multiobjective programming problem. Let $\eta: R \times R \rightarrow R$ be defined by

$$
\eta(x, \bar{x})=e^{x}-e^{\bar{x}} .
$$

Now, we construct the associated $\eta$-approximated vector optimization problem $\left(\operatorname{VP} 2_{\eta}(\bar{x}, \bar{\xi}, \bar{\zeta})\right)$ by transforming at $\bar{x}$ both the objective function $f$ and the constraint function $g$. Thus, we obtain the following vector optimization problem:

$$
\begin{gathered}
\left(e^{x+1}-e, \bar{\xi}_{2}\left(e^{x}-1\right)\right) \rightarrow \min , \\
1-e^{x} \leqq 0, \\
-2+\bar{\zeta}_{2}\left(e^{x}-1\right) \leqq 0,
\end{gathered}
$$

where $\bar{\xi}$ and $\bar{\zeta}$ are Clarke's generalized gradients of $f$ and $g$ at $\bar{x}$, that is,

$$
\bar{\xi} \in \partial f(\bar{x})=\left\{\left[e, \bar{\xi}_{2}\right]^{T} \mid-1 \leqq \bar{\xi}_{2} \leqq 1\right\} \quad \text { and } \quad \bar{\zeta} \in \partial g(\bar{x})=\left\{\left[1, \bar{\zeta}_{2}\right]^{T} \mid-1 \leqq \bar{\zeta}_{2} \leqq 1\right\} \text {, }
$$

satisfying the generalized Karush-Kuhn-Tucker necessary optimality conditions (2.3)-(2.5). Since $\eta$ satisfies the condition $\eta(\bar{x}, \bar{x})=0$, then, by Theorem 3.1, $\bar{x}=0$ is also a Pareto optimal solution in the associated $\eta$-approximated vector optimization problem $\left(\operatorname{VP}_{\eta}(\bar{x}, \bar{\xi}, \bar{\zeta})\right)$ defined above. In contrast, to prove by Theorem 3.6 that $\bar{x}$, being a (weak) Pareto optimal solution in $\left(\operatorname{VP}_{\eta}(\bar{x}, \bar{\xi}, \bar{\zeta})\right)$, is also a (weak) Pareto optimal solution in the original multiobjective programming problem (VP), we need to show that $f$ and $g$ are $V$-invex at $\bar{x}$ on $D$ with respect to the same $\eta$ satisfying the condition $\eta(\bar{x}, \bar{x})=0$. If we set, for example,

$$
\begin{aligned}
& \alpha_{1}(x, \bar{x})=1, \quad \alpha_{2}(x, \bar{x})= \begin{cases}1 & \text { if } x=0, \\
\frac{\arctan x}{e^{x}-e^{\bar{x}}} & \text { if } 0<x \leqq 2,\end{cases} \\
& \beta_{1}(x, \bar{x})=\left\{\begin{array}{lll}
1 & \text { if } x=0, \\
\frac{x}{e^{x}-e^{\bar{x}}} & \text { if } 0<x \leqq 2, & \beta_{2}(x, \bar{x})=1 .
\end{array}\right.
\end{aligned}
$$

then, by Definition 2.4, $f$ is $V$-invex at $\bar{x}$ on $D$ with respect to $\eta$ and $\alpha=\left(\alpha_{1}, \alpha_{2}\right)$ and $g$ is $V$-invex at $\bar{x}$ on $D$ with respect to the same function $\eta$ and with respect to $\beta=\left(\beta_{1}, \beta_{2}\right)$.

It is not difficult to see that any $\eta$-approximated vector optimization problem $\left(\operatorname{VP} 2_{\eta}(\bar{x}, \bar{\xi}, \bar{\zeta})\right)$ belonging to a family of this vector optimization problem is easier to solve than the original nonsmooth multiobjective programming problem (VP2).

REMARK 3.8. Note that each $\eta$-approximated vector optimization problem $\left(\operatorname{VP} 2_{\eta}(\bar{x}, \bar{\xi}, \bar{\zeta})\right)$ constructed in the $\eta$-approximation method for the nondifferentiable multiobjective programming problem (VP2) considered in Example 3.7 is smooth. Thus, the $\eta$-approximation method allows the characterization of solvability of a nondifferentiable multiobjective programming problem (VP) by the help of a smooth vector optimization problem. This property is useful from a practical point of view. 
REMARK 3.9. It is not difficult to see that if the function $\eta$, with respect to which all functions constituting the considered nonsmooth multiobjective programming problem (VP) are $V$-invex, is linear with respect to the first component, then each $\eta$ approximated vector optimization problem constructed in the $\eta$-approximation method is a linear vector optimization problem.

Now, we give an example of a nonsmooth multiobjective programming problem for which its $\eta$-approximated vector optimization problems constructed in the $\eta$ approximation method are linear.

EXAMPLE 3.10. We consider the following nonlinear nonsmooth multiobjective programming problem:

$$
\begin{gathered}
f(x)=\left(\arctan |e x|, x \ln \left(\left|x-\frac{1}{2} e\right|+\frac{1}{2} e\right)\right) \rightarrow \min \\
g(x)=\ln ^{2}(x+1)-\ln (x+1) \leqq 0 \\
X=\{x \in R \mid x>-1\}
\end{gathered}
$$

Note that $D=\{x \in R \mid 0 \leqq x \leqq e-1\}$ and $\bar{x}=0$ is a Pareto optimal point in the considered nonlinear multiobjective programming problem. Let $\eta: X \times X \rightarrow R$ be defined as follows:

$$
\eta(x, \bar{x})=\frac{1}{e}(x-\bar{x})
$$

We construct the $\eta$-approximated vector optimization problem $\left(\operatorname{VP} 3_{\eta}(\bar{x}, \bar{\xi}, \bar{\zeta})\right)$ associated with (VP3) as follows:

$$
\begin{gathered}
\left(\frac{1}{e} \bar{\xi}_{1} x, \frac{1}{e} x\right) \rightarrow \min \\
-\frac{1}{e} x \leqq 0
\end{gathered}
$$

where $\bar{\xi}$ and $\bar{\zeta}$ are Clarke's generalized gradients of $f$ and $g$ at $\bar{x}$, that is, $\bar{\xi} \in \partial f(\bar{x})=\left\{\left[\bar{\xi}_{1}, 1\right]^{T} \mid-e \leqq \bar{\xi}_{1} \leqq e\right\}$ and $\bar{\zeta}=-1$, satisfying the generalized KarushKuhn-Tucker necessary optimality conditions (2.3)-(2.5). Since $\eta$ satisfies the condition $\eta(\bar{x}, \bar{x})=0$, then, by Theorem $3.1, \bar{x}=0$ is also a Pareto optimal solution in $\left(\operatorname{VP} 3_{\eta}(\bar{x}, \bar{\xi}, \bar{\zeta})\right)$.

In contrast, we consider any $\eta$-approximated vector optimization problem $\left(\operatorname{VP}_{\eta}(\bar{x}, \bar{\xi}, \bar{\zeta})\right)$ defined above. To prove, by Theorem 3.6, that $\bar{x}$, being a (weak) Pareto optimal solution in $\left(\operatorname{VP} 3_{\eta}(\bar{x}, \bar{\xi}, \bar{\zeta})\right)$, is also a (weak) Pareto optimal solution in the original multiobjective programming problem (VP3) it should be proved that $f$ and $g$ are $V$-invex at $\bar{x}$ on $D$ with respect to the same $\eta$ satisfying the condition 
$\eta(\bar{x}, \bar{x})=0$. If we set, for example,

$$
\begin{aligned}
& \alpha_{1}(x, \bar{x})=\left\{\begin{array}{ll}
\frac{1}{\arctan e x} & \text { if } x=0, \\
x & \text { if } 0<x \leqq e-1,
\end{array} \quad \alpha_{2}(x, \bar{x})=e \ln \left(\left|x-\frac{1}{2} e\right|+\frac{1}{2} e\right),\right. \\
& \beta(x, \bar{x})= \begin{cases}\frac{e}{\frac{e}{x}\left[\ln (x+1)-\ln ^{2}(x+1)\right]} & \text { if } 0<0 \vee x<e-1 .\end{cases}
\end{aligned}
$$

then, by Definition 2.4, $f$ is $V$-invex at $\bar{x}$ on $D$ with respect to $\eta$ and $\alpha=$ $\left(\alpha_{1}, \alpha_{2}\right)$ and $g$ is $V$-invex at $\bar{x}$ on $D$ with respect to the same function $\eta$ and with respect to $\beta=\left(\beta_{1}, \beta_{2}\right)$. Note that each $\eta$-approximated vector optimization problem $\left(\mathrm{VP} 3_{\eta}(\bar{x}, \bar{\xi}, \bar{\zeta})\right)$ constructed for the considered nondifferentiable multiobjective programming problem (VP3) is a smooth linear vector optimization problem, since the function $\eta$, with respect to all functions constituting the considered multiobjective programming problem (VP3), is linear with respect to the first component. This property of the $\eta$-approximation method is also useful from a practical point of view.

REMARK 3.11. The assumption that a function $\eta$ satisfies the condition $\eta(\bar{x}, \bar{x})=0$ is essential to confirm the equivalence between the vector optimization problems (VP) and $\left(\operatorname{VP}_{\eta}(\bar{x}, \bar{\xi}, \bar{\zeta})\right)$ in the sense discussed in the paper. In the example below, we show that in the case when this condition does not hold then there is no equivalence between vector optimization problems $(\mathrm{VP})$ and $\left(\mathrm{VP}_{\eta}(\bar{x}, \bar{\xi}, \bar{\zeta})\right)$.

EXAMPLE 3.12. We consider the following nonlinear multiobjective programming problem:

$$
\begin{aligned}
f\left(x_{1}, x_{2}\right) & =\left(f_{1}\left(x_{1}, x_{2}\right), f_{2}\left(x_{1}, x_{2}\right)\right) \rightarrow \min , \\
g_{1}\left(x_{1}, x_{2}\right) & =e^{x_{1}-1}-2 \ln x_{1}-x_{2} \leqq 0 \\
g_{2}\left(x_{1}, x_{2}\right) & =-\ln x_{1} \leqq 0 \\
X & =\left\{\left(x_{1}, x_{2}\right) \in R^{2} \mid x_{1}>0\right\}
\end{aligned}
$$

where

$$
\begin{aligned}
& f_{1}\left(x_{1}, x_{2}\right)= \begin{cases}x_{1}-2+x_{2} & \text { if } 0<x_{1} \leqq 2, \\
2\left(x_{1}-2\right)+x_{2} & \text { if } x_{1}>2,\end{cases} \\
& f_{2}\left(x_{1}, x_{2}\right)= \begin{cases}\frac{1}{2}\left(x_{1}-1\right)+\frac{1}{2} x_{2} & \text { if } 0<x_{1} \leqq 1, \\
\frac{1}{4}\left(x_{1}-1\right)+\frac{1}{2} x_{2} & \text { if } x_{1}>1 .\end{cases}
\end{aligned}
$$

Note that $D=\left\{\left(x_{1}, x_{2}\right) \in X \mid x_{1} \geqq 1 \wedge x_{2} \geqq 1\right\}$ and $\bar{x}=(1,1)$ is a Pareto optimal point in the considered multiobjective programming problem. Furthermore, $f$ is $V$ invex at $\bar{x}$ on $D$ with respect to $\eta$ and $\alpha=\left(\alpha_{1}, \alpha_{2}\right)$ and $g$ is $V$-invex with respect to the same function $\eta$ and with respect to $\beta=\left(\beta_{1}, \beta_{2}\right)$ defined by

$$
\begin{aligned}
\eta(x, \bar{x}) & =\left[\begin{array}{l}
x_{1}+\bar{x}_{1}+1 \\
x_{2}+\bar{x}_{2}-5
\end{array}\right], \\
\alpha_{1}(x, \bar{x})=1, \quad \alpha_{2}(x, \bar{x}) & =\frac{1}{2}, \quad \beta_{1}(x, \bar{x})=1, \quad \beta_{2}(x, \bar{x})=1 .
\end{aligned}
$$


It is not difficult to see that the condition $\eta(\bar{x}, \bar{x})=0$ is not fulfilled by the function $\eta$ defined above. For the considered nonlinear multiobjective programming problem, we construct its associated $\eta$-approximated vector optimization problem $\left(\operatorname{VP} 4_{\eta}(\bar{x}, \bar{\xi}, \bar{\zeta})\right)$. Thus, we obtain

$$
\begin{gathered}
\left(x_{1}+x_{2}-2, \bar{\xi}_{21}\left(x_{1}+2\right)+\frac{1}{2} x_{2}-\frac{3}{2}\right) \rightarrow \min \\
-x_{1}-x_{2}+2 \leqq 0 \\
-x_{1}-4 \leqq 0
\end{gathered}
$$

where $\bar{\xi}$ and $\bar{\zeta}$ are Clarke's generalized gradients of $f$ and $g$ at $\bar{x}$, that is,

$$
\bar{\xi} \in \partial f(\bar{x})=\left\{\left[\begin{array}{cc}
1 & 1 \\
\bar{\xi}_{21} & \frac{1}{2}
\end{array}\right] \mid \frac{1}{4} \leqq \bar{\xi}_{21} \leqq \frac{1}{2}\right\} \quad \text { and } \quad \bar{\zeta} \in \partial g(\bar{x})=\left[\begin{array}{cc}
-1 & -1 \\
-1 & 0
\end{array}\right]
$$

satisfying the generalized Karush-Kuhn-Tucker necessary optimality conditions (2.3)-(2.5). Therefore, $\bar{x}=(1,1)$ is not a Pareto optimal point in the above vector optimization problem. Thus, the considered multiobjective programming problem (VP4) and its associated $\eta$-approximated vector optimization problems $\left(\mathrm{VP}_{\eta}(\bar{x}, \bar{\xi}, \bar{\zeta})\right)$ are not equivalent in the sense discussed in this paper.

\section{Conclusion}

In this paper we have introduced a new approach for solving a nonlinear (nonconvex) nondifferentiable multiobjective programming problem. The formulation of the introduced $\eta$-approximated vector optimization problem requires the Lagrange multipliers of the original multiobjective programming problem. Thus, apparently one cannot compute the $\eta$-approximated vector optimization problem without first computing the original multiobjective programming problem. This follows from the formulation of the introduced $\eta$-approximation approach, since we need both a point $\bar{x}$, which is expected to be optimal, and Clarke's generalized gradients $\bar{\xi}$ and $\bar{\zeta}$ of the objective function $f$ and the constraint function $g$ at $\bar{x}$, respectively. Then, a family of $\eta$-approximated vector optimization problems can be constructed at such a selected point. In general, we obtain a simpler vector optimization problem which is easier to solve than the original nonlinear nondifferentiable multiobjective programming problem. It turns out that the $\eta$-approximation method affords possibilities for characterizing solvability of a nondifferentiable nonconvex multiobjective programming problem with the help of the linear (or convex) smooth vector optimization problems (see Examples 3.7 and 3.10 and Remarks 3.8 and 3.9). Moreover, as follows from the formulation of an $\eta$-approximated vector optimization problem and the definition of $V$-invexity, there may exist more than one associated $\eta$ approximated vector optimization problem equivalent to the considered multiobjective programming problem (VP). These properties are also useful from a practical point of view. 


\section{References}

[1] T. Antczak, "A new approach to multiobjective programming with a modified objective function", J. Global Optim. 27 (2003) 485-495.

[2] T. Antczak, "An $\eta$-approximation method in nonlinear vector optimization", Nonlinear Anal. 63 (2005) 225-236.

[3] T. Antczak, "Saddle points criteria and duality in multiobjective programming via an $\eta$ approximation method", J. Aust. Math. Soc. B 47 (2005) 155-172.

[4] T. Antczak, "An $\eta$-approximation approach in nonlinear vector optimization with univex functions”, Asia-Pac. J. Oper. Res. 23 (2006) 525-542.

[5] T. Antczak, "Optimality and duality for nonsmooth multiobjective programming problems with $V$-r-invexity", J. Global Optim. to appear.

[6] F. H. Clarke, Optimization and nonsmooth analysis, in Canadian Mathematical Society Series of Monographs and Advanced Texts (John Wiley \& Sons, New York, 1983).

[7] B. D. Craven, "Invex functions and constrained local minima", Bull. Austral. Math. Soc. 24 (1981) 357-366.

[8] B. D. Craven, "Quasimin and quasisaddlepoint for vector optimization", Numer. Funct. Anal. Optim. 11 (1990) 45-54.

[9] R. R. Egudo and M. A. Hanson, "On sufficiency of Kuhn-Tucker conditions in nonsmooth multiobjective programming", FSU Technical Report No. M-888, 1993.

[10] G. Giorgi and A. Guerraggio, "The notion of invexity in vector optimization: smooth and nonsmooth case", in Generalized convexity, generalized monotonicity, Proc. Fifth Symp. on Generalized convexity of Luminy, France (eds. J. P. Crouzeix, J. E. Martinez-Legaz and M. Volle), (Kluwer Academic, Dordrecht, 1997).

[11] M. A. Hanson, "On sufficiency of the Kuhn-Tucker conditions", J. Math. Anal. Appl. 80 (1981) $545-550$.

[12] V. Jeyakumar and B. Mond, "On generalized convex mathematical programming", J. Aust. Math. Soc. B 34 (1992) 43-53.

[13] D. S. Kim and S. Schaible, "Optimality and duality for invex nonsmooth multiobjective programming problems", Optimization 53 (2004) 165-176.

[14] G. M. Lee, "Nonsmooth invexity in multiobjective programming", J. Inf. Optim. Sci. 15 (1994) $127-136$.

[15] D. T. Luc, Theory of vector optimization, Volume 319 of Lecture Notes in Economics and Mathematical Systems (Springer, Berlin, 1989).

[16] T. W. Reiland, "Nonsmooth invexity", Bull. Austral. Math. Soc. 42 (1990) 437-446.

[17] P. Ruiz-Canales and A. Rufián-Lizana, "A characterization of weakly efficient points", Math. Program. 68 (1995) 205-212.

[18] Y. Sawaragi, H. Nakayama and T. Taninio, Theory of multiobjective optimization (Academic Press, Orlando, FL, 1985).

[19] C. Singh, "Optimality conditions in multiobjective differentiable programming", J. Optim. Theory Appl. $\mathbf{5 3}$ (1987) 115-123.

[20] R. E. Steuer, Multiple criteria optimization: theory, computation, and application (Wiley, New York, 1986).

[21] T. Taninio, "Stability and sensitivity analysis in convex vector optimization", SIAM J. Control Optim. 26 (1988) 521-536.

[22] T. Weir, B. Mond and B. D. Craven, "On duality for weakly minimized vector valued optimization problems”, Optimization 17 (1986) 711-721. 